\title{
Methods for Interpreting Partitioning and Fate of Petroleum Hydrocarbons in a Sea Ice Environment
}

\author{
Durell S. Desmond ${ }^{*}$, Diana Saltymakova ${ }^{1}$, \\ Odile Crabeck ${ }^{2,3}$, Georg Schreckenbach ${ }^{1}$, James D. Xidos ${ }^{1}$, \\ David G. Barber ${ }^{1}$, Dustin Isleifson ${ }^{1}$, and Gary A. Stern ${ }^{1 *}$ \\ ${ }^{1}$ University of Manitoba, Winnipeg, Manitoba, R3T 2N2, Canada \\ 2Laboratoire de Glaciologie, Université Libre de Bruxelles, Bruxelles, 99131, Belgium \\ 3Unité d'Océanographie Chimique, Freshwater and Oceanic sCience Unit reSearch \\ (FOCUS), Université de Liège, Liège, Belgium \\ *Email: umdesmod@myumanitoba.ca \\ *Email: Gary.Stern@umanitoba.ca
}

Table S 1 Table of identified compounds in the crude oil composition, their quantifiers, and recovery standards

\begin{tabular}{|c|c|c|c|c|}
\hline Compounds & $\begin{array}{l}\text { Target } \\
\text { Ion }\end{array}$ & Quantifier & $\begin{array}{l}\text { \# of } \\
\text { comp. }\end{array}$ & Recovery std. \\
\hline \multicolumn{5}{|c|}{ Saturated compounds } \\
\hline Alkanes $\mathrm{C}_{11}-\mathrm{C}_{36}$ & 71.0861 & N-alkanes ${ }^{1}$ & 26 & $\begin{array}{l}\text { Closest d- } \\
\text { alkane }\end{array}$ \\
\hline$*$ Tricyclic Terpane $\mathrm{C}_{20}-\mathrm{C}_{23}$ & 191.18 & $\begin{array}{c}\text { 17a(H)-22,29,30 - } \\
\text { Trisnorhopane }\end{array}$ & 4 & $\mathrm{D}_{42}$ Eicosane \\
\hline$*$ Tricyclic Terpane $\mathrm{C}_{24}-\mathrm{C}_{26}$ & 191.18 & $\begin{array}{c}\text { 17a }(\mathrm{H})-22,29,30- \\
\text { Trisnorhopan }\end{array}$ & 4 & $\mathrm{D}_{50}$ Tetracosane \\
\hline${ }^{*}$ Tricyclic Terpane $\mathrm{C}^{28}-\mathrm{C}_{31}$ & 191.18 & $\begin{array}{c}17 \mathrm{a}(\mathrm{H})-22,29,30- \\
\text { Trisnorhopane }^{3}\end{array}$ & 8 & $\mathrm{D}_{58}$ Octacosane \\
\hline${ }^{*}$ Tetracyclic Terpane $\mathrm{C}_{24}$ & 191.18 & $\begin{array}{c}\text { 17a }(\mathrm{H})-22,29,30- \\
\text { Trisnorhopane }\end{array}$ & 1 & $\mathrm{D}_{50}$ Tetracosane \\
\hline${ }^{*}$ Hopanes $\mathrm{C}_{27}-\mathrm{C}_{30}$ & 191.18 & Hopanes $^{3}$ & 6 & $\mathrm{D}_{58}$ Octacosane \\
\hline $\begin{array}{l}* 18 \mathrm{a}(\mathrm{H})-22,29,30- \\
\text { Trisnorneohopane }\end{array}$ & 191.18 & $\begin{array}{c}17 \mathrm{a}(\mathrm{H})-22,29,30- \\
\text { Trisnorhopane }^{3}\end{array}$ & 1 & $\mathrm{D}_{58}$ Octacosane \\
\hline *17a(H),21(b)-23 S/R-Homohopane & 191.18 & $\begin{array}{c}17 \mathrm{a}(\mathrm{H}), 21(\mathrm{~b})-22 \mathrm{~S} / \mathrm{R}- \\
\text { Homohopane }^{3}\end{array}$ & 2 & $\mathrm{D}_{58}$ Octacosane \\
\hline$*$ Diasteranes $\mathrm{C}_{21}-\mathrm{C}_{26}$ & 217.1956 & $\alpha \alpha \alpha 20$ S-Cholestane ${ }^{3}$ & 6 & $\mathrm{D}_{50}$ Tetracosane \\
\hline$*$ Regular sterane $\mathrm{C}_{21}$ & 217.1956 & $\alpha \alpha \alpha$ 20S-Cholestane ${ }^{3}$ & 1 & $\mathrm{D}_{50}$ Tetracosane \\
\hline *Iso-steranes $\mathrm{C}_{21}-\mathrm{C}_{23}$ & 217.1956 & $\alpha \beta \beta$ 20S-Cholestane ${ }^{3}$ & 5 & $\mathrm{D}_{50}$ Tetracosane \\
\hline
\end{tabular}

\footnotetext{
${ }^{1}$ n-alkanes (mix of tree SPEX standards: $\mathrm{C}_{11}-\mathrm{C}_{18}$, odd $\mathrm{C}_{15}-\mathrm{C}_{35}$, even $\mathrm{C}_{16}-\mathrm{C}_{36}$ );

2 hopanes and steranes (chiron, 12 mix)
} 


\begin{tabular}{|c|c|c|c|c|}
\hline Compounds & $\begin{array}{l}\text { Target } \\
\text { Ion }\end{array}$ & Quantifier & $\begin{array}{l}\text { \# of } \\
\text { comp. }\end{array}$ & Recovery std. \\
\hline *Diasteranes $\mathrm{C}_{27}-\mathrm{C}_{29}$ & 217.1956 & $\alpha \alpha \alpha$ 20S-Cholestane ${ }^{3}$ & 9 & $\mathrm{D}_{58}$ Octacosane \\
\hline$*$ Regular and iso-steranes $\mathrm{C}_{27}-\mathrm{C}_{29}$ & 217.1956 & $\begin{array}{l}\text { The closest regular or iso- } \\
\text { sterane from steranes std }\end{array}$ & 9 & $\mathrm{D}_{58}$ Octacosane \\
\hline \multicolumn{5}{|c|}{ Aromatic compounds } \\
\hline Naphthalene & 128.0626 & Naphthalene ${ }^{3}$ & 1 & $\mathrm{D}_{8}$ Napthalene \\
\hline 1; 2 -Methylnaphthalenes & 142.0783 & 1-Methylnaphthalene ${ }^{4}$ & 2 & $\begin{array}{c}\mathrm{D}_{8} \\
\text { Acenaphthylen } \\
\mathrm{e}\end{array}$ \\
\hline Dimethylnaphthalenes & 156.0939 & 1,6-Dimethylnaphthalene ${ }^{6}$ & 8 & $\begin{array}{c}\mathrm{D}_{10} \\
\text { Acenaphthene }\end{array}$ \\
\hline Trimethylnaphthalenes & 170.1096 & 2,3,5-Trimethylnaphthalene ${ }^{6}$ & 7 & $\mathrm{D}_{10}$ Fluorene \\
\hline Tetramethylnaphthalenes & 184.1252 & 2,3,5-Trimethylnaphthalene ${ }^{6}$ & 9 & $\mathrm{D}_{10}$ Fluorene \\
\hline Pentamethylnaphthalenes & 198.1409 & 2,3,5-Trimethylnaphthalene ${ }^{6}$ & 4 & $\begin{array}{c}\mathrm{D}_{10} \\
\text { Phenanthrene } \\
\end{array}$ \\
\hline \multicolumn{5}{|c|}{ Surrogate compounds } \\
\hline Deuterated PAHs & & $\mathrm{D}_{8}, \mathrm{D}_{10}, \mathrm{D}_{12}^{5}$ & 16 & d- PAHs \\
\hline Deuterated Alkanes & 66.1256 & $\begin{array}{c}\mathrm{D}_{26}, \mathrm{D}_{34}, \mathrm{D}_{42}, \mathrm{D}_{50}, \mathrm{D}_{58}, \mathrm{D}_{66}, \\
\mathrm{D}_{72}{ }^{6}\end{array}$ & 7 & d-alkanes \\
\hline
\end{tabular}

*- analyzed on Triple Quadrupole GC-MS

${ }^{3}$ PAHs mix (CV calibration mix \# 5, Restek, 16 mix)

${ }^{4}$ PAH Dibenzothiophene (and alkylated homologues) mixture (Chiron, $20 \mathrm{mix}$ )

${ }^{5}$ Deuterated PAH $\left(\mathrm{D}_{8}, \mathrm{D}_{10}, \mathrm{D}_{12}\right.$, Wellington Laboratories, 8 mix $)$

${ }^{6}$ n-alkane $\left(D_{26}-D_{72}\right.$, Fisherbrand, 7 mix $)$ 
Table S 2 Multiple reaction monitoring mass spectrometry method for steranes and tepanes

\begin{tabular}{|c|c|c|c|c|c|}
\hline $\begin{array}{c}\text { Compound } \\
\text { name }\end{array}$ & Precursor & Product & Dwell & $\begin{array}{c}\text { CE } \\
(\mathrm{eV})\end{array}$ & Type \\
\hline \multirow{3}{*}{$\begin{array}{c}\text { Regular and } \\
\text { diastaranes }\end{array}$} & 217 & 93 & 30 & 24 & Qualifier \\
\hline & 217 & 121 & 30 & 12 & Quantifier \\
\hline & 217 & 135 & 30 & 10 & Qualifier \\
\hline \multirow{3}{*}{$\alpha \beta \beta$ Steranes } & 218 & 93 & 30 & 24 & Qualifier \\
\hline & 218 & 161 & 30 & 14 & Qualifier \\
\hline & 218 & 175 & 30 & 12 & Quantifier \\
\hline$\beta \alpha$ Steranes & 259 & 95 & 30 & 20 & Qualifier \\
\hline Steranes $\mathrm{C}_{26}$ & 358 & 217 & 30 & 20 & Qualifier \\
\hline Steranes $\mathrm{C}_{27}$ & 372 & 217 & 30 & 20 & Qualifier \\
\hline Steranes $\mathrm{C}_{28}$ & 386 & 217 & 30 & 20 & Qualifier \\
\hline Steranes $\mathrm{C}_{29}$ & 400 & 217 & 30 & 20 & Qualifier \\
\hline \multirow{5}{*}{ Terpanes } & 191 & 107 & 30 & 16 & Qualifier \\
\hline & 191 & 95 & 30 & 12 & Quantifier \\
\hline & 177 & 93 & 30 & 20 & Qualifier \\
\hline & 177 & 107 & 30 & 12 & Qualifier \\
\hline & 177 & 121 & 30 & 10 & Qualifier \\
\hline
\end{tabular}

